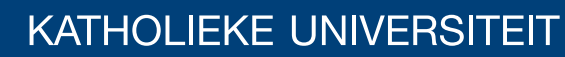 \\ LEUVEN
}

\section{Faculty of Business and Economics}

\section{UDFWFDORQURQVIRUDIGRFNDWJ QP HQVSLREOP

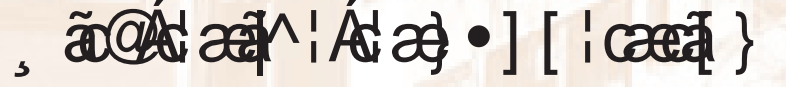

/ RUKL\%HJ KP DQDDQG55 RHOO HXV

DEPARTMENT OF DECISION SCIENCES AND INFORMATION MANAGEMENT (KBI) 


\title{
Practical solutions for a dock assignment problem with trailer transportation
}

\author{
L. Berghman, R. Leus \\ ORSTAT, K.U.Leuven, Naamsestraat 69, 3000 Leuven, Belgium \\ \{ Lotte.Berghman ; Roel.Leus \}@econ.kuleuven.be
}

\begin{abstract}
We study a distribution warehouse in which trailers need to be assigned to docks for loading or unloading. A parking lot is used as a buffer zone and transportation between the parking lot and the docks is performed by auxiliary resources called terminal tractors. Each incoming trailer has a known arrival time and each outgoing trailer a desired departure time. The primary objective is to produce a docking schedule such that the weighted sum of the number of late outgoing trailers and the tardiness of these trailers is minimized; the secondary objective is to minimize the weighted completion time of all trailers, both incoming and outgoing. The purpose of this paper is to produce high-quality solutions to large instances that are comparable to a real-life case. We implement several heuristic algorithms: truncated branch and bound, beam search and tabu search. Lagrangian relaxation is embedded in the algorithms for constructing an initial solution and for computing lower bounds. The different solution frameworks are compared via extensive computational experiments.
\end{abstract}

Keywords: dock assignment, multicriteria scheduling, branch and bound, beam search, Lagrangian relaxation, tabu search.

\section{Introduction}

We study a distribution warehouse with several docks, where incoming trailers are unloaded after they arrive and where outgoing trailers are loaded before they leave. Each dock can be occupied by at most one trailer at any moment in time. The site also contains a parking lot, which serves as a buffer area where trailers are temporarily parked. We distinguish between three types of trailers. First of all, we have the coupled trailers. These trailers arrive at the parking lot at a known arrival time (a release date) and are brought to the dock by the trucker, who waits until the load or unload activity is completed to take the trailer away. A desired latest departure time (a due date) is specified for each of these trailers to avoid having truckers wait for excessive time at the plant. A second type of trailers are the uncoupled incoming trailers that are to be unloaded. These trailers also have a known arrival time (a release date) but no restrictive due date. The third set of trailers are the uncoupled outgoing trailers to be loaded, which are available at the parking area from the outset. These trailers have a due date, since these trailers need to be transported to clients after they are loaded. Uncoupled trailers (both incoming and outgoing) are dropped off by a trucker at the parking lot and afterwards transferred to a dock by one of the terminal tractors, which are tractors designed for use in ports, terminals and heavy industry. After unloading or loading at the dock, the uncoupled trailer is moved back to the parking lot by a tractor, where it will be picked up by a trucker later on. 
The described dock assignment problem is modeled after a case encountered at Toyota Parts Center Europe (TPCE), a Toyota warehouse in Diest, Belgium. The stated assumptions closely adhere to this practical situation. In an earlier paper (Berghman et al., to appear), we have explored the possibility of finding optimal solutions for small instances by means of integer (linear) programming (IP). In that study, the due dates were treated as strict deadlines. After discussion with the management of the site, however, it turns out that these latest departure times are better modeled as due dates: the existence of a feasible schedule meeting all due dates is not guaranteed, but satisfying them is our primary objective. Minimization of the waiting times of the trailers is the secondary objective.

The purpose of this paper is to produce practical solutions to the dock assignment problem. The practical character of the work resides in the hierarchical use of two objectives, which is in line with the requirements of the management. Furthermore, we ambition to produce high-quality solutions to realistic instances. Our results in Section 4 will indicate that large instances cannot be solved to guaranteed optimality within reasonable running times, and we will therefore resort to the development of heuristic procedures. The contributions of this text are fourfold: (1) we cast the practical problem setting into a hierarchical bi-objective optimization problem; (2) we present an IP formulation for this problem; (3) we propose different heuristic algorithms; and (4) we investigate how Lagrangian relaxation can lead to an initial solution and lower bounds. Our computational experiments will show that the best solutions are obtained by a hybrid of two algorithms.

The remainder of this article is structured as follows. Section 2 provides a brief literature survey on the related topics of dock scheduling, multicriteria scheduling and flexible flow-shop scheduling. In Section 3, some definitions and a formal problem statement are presented. An IP formulation will be proposed in Section 4. Section 5 explains how schedules will be represented in our solution procedures. Subsequently, a branch-and-bound (B\&B) algorithm, a beam search algorithm and a tabu search algorithm will be represented in Sections 6,7 and 8 respectively. An overview of our computational results is given in Section 9 and we round off the article with a summary and some conclusions in Section 10.

\section{Literature review}

In this section, we provide a brief review of the recent relevant work in different fields. First, we describe the literature on dock scheduling including cross docking (Section 2.1). Secondly, the literature on multicriteria scheduling is surveyed in Section 2.2 and finally, a brief overview is included of the literature on flexible flow-shop scheduling (Section 2.3).

\subsection{Dock scheduling}

The problem presented in this paper is a dock assignment problem: trailers are assigned to docks for a limited period of time for loading or unloading activities. The storage capacity of the warehouse is not restricted and there are no links between incoming and outgoing shipments. All goods stay at least one night in the warehouse, such that a product that is unloaded at day $X$ will be forwarded at day $X+1$ at the earliest. We are not aware of existing scientific papers with exactly the same setup. 
Two related settings with trailer scheduling that have received attention in the recent literature are cross docking, and operations planning at container terminals. According to $\mathrm{Yu}$ and Egbelu (2008), "Cross docking is a warehouse management concept in which items delivered to a warehouse by incoming trucks are immediately sorted out, reorganized based on customer demands, routed and loaded into outgoing trucks for delivery to customers without the items being actually held in inventory at the warehouse." The advantages are faster deliveries, lower inventory costs and a reduction of the warehouse space requirement. A comprehensive overview of different variations and the available literature can be found in Boysen and Fliedner (2010). The truck-dock assignment problem examines the scheduling of a set of trailers at docks over time (Miao et al. 2009). A number of area-specific constraints are added in order to link the incoming and outgoing shipments (see Boysen et al. 2010) or to model the operations within the cross dock (see Miao et al. 2009). Heuristics are often used to solve realistic instances (see, e.g., Soltani and Sadjadi 2010; Boloori Arabani et al. 2011; Alpan et al. to appear). The dock assignment problem is similar to the truck-dock assignment problem in cross docking, but in our case there is no explicit restriction on the warehouse capacity and the incoming and outgoing shipments are unrelated, so there are no precedence constraints between different trailers.

Böse et al. (2000) describe the main logistic processes in seaport container terminals and propose evolutionary algorithms for optimization. Bish et al. (2001) and Bish et al. (2005) concentrate on the transportation of containers from a ship to a yard using a fleet of vehicles. Since the authors focus on the performance for large instances, heuristics are put forward. Our problem also covers the transportation of trailers (containers), but we have no additional constraints such as cranes that cannot cross.

\subsection{Multicriteria scheduling}

When a schedule's quality is evaluated on multiple performance criteria, in most cases there will be no schedule that achieves the optimal value for all criteria simultaneously. Consequently, sacrifices will need to be made with regard to the performance for at least one of the conflicting criteria. Chen and Bulfin (1993) present an overview of such multicriteria scheduling problems on a single machine. More general shop layouts are discussed in Hoogeveen (2005) and T'kindt and Billaut (2006).

A common approach to dealing with multicriteria scheduling is to aggregate the different criteria into one composite objective function, a process that is often called scalarization or simultaneous optimization (Baker and Smith, 2003; Hoogeveen, 2005). If one criterion is dominant, however, the decision maker will prefer to first distinguish the set of all schedules that are optimal with respect to the primary objective and then search within this set of schedules for one that is best for a secondary objective (Pinedo, 2008). This approach is called hierarchical or lexicographic optimization and is an example of a non-scalarizing method (Sarin and Hariharan, 2000; T'kindt et al., 2003).

\subsection{Flexible flow-shop scheduling}

The dock assignment problem studied in this paper can be modeled as a flexible flow shop. In a flexible flow shop, also called hybrid or multi-processor flow shop, at least one stage consists 
of parallel machines. The terminal tractors in this paper can be modeled as machines rather than transporters, especially since the time it takes the tractors to convey a trailer between the docks and the parking lot is essentially independent of the distance (see Section 3). In this way, the transportation activities become stages one and three of a flexible flow shop, and the load/unload activities constitute the second stage. In our problem, the same set of identical machines (the tractors) executes both the first and the third stage of the uncoupled trailers, while the second stage of all trailers takes place on another set of identical machines (the docks). None of these machines are needed for the first and the third stage of the coupled trailers.

Linn and Zhang (1999), Vignier et al. (1999) and Ribas et al. (2010) all provide surveys of the flexible flow-shop literature. Most studies deal with two-stage flow shops with parallel machines either in the first or in the second stage, but not in both. Many research articles related to flexible flow-shop scheduling are available, but most of these do not handle unequal ready times. Both approximation (see, e.g., Tang and Xuan 2006; Nichi et al. 2010) and optimal approaches (for instance Kis and Pesch 2005; Haouari et al. 2006) have been published.

A limited number of articles propose solution procedures for flow-shop scheduling with release times. Moursli and Pochet (2000) introduce a B\&B algorithm for makespan minimization that produces high-quality results even when it is truncated after a few minutes of computation time. Gupta et al. (2002) generalize well-known heuristic approaches and present constructive algorithms based on job insertion techniques and iterative algorithms based on local search. Paternina-Arboleda et al. (2008) propose a heuristic for makespan minimization that focuses on the identification and exploitation of the bottleneck stage.

\section{Definitions and problem statement}

We pointed out in Section 2.3 that three activities are performed for each trailer (corresponding to three stages in a flexible flow shop): the movement from the parking lot to a dock, the loading or unloading task and the transportation back to the parking area. The decisions to be made are the timing of each of the three activities, the choice of the tractor for stages one and three of the uncoupled trailers, and the choice of the dock for stage two of all trailers. The load/unload times may differ between jobs but do not depend on the dock. The transportation activities are modeled as having a constant duration of one time unit, because the time to follow the safety instructions is large compared to the actual transportation time.

In this article, we will use the terms 'job' and 'trailer' interchangeably, and the terms 'task' and 'activity' are also equivalent. The set $J$ contains all jobs, with $|J|=n$, and $T$ is the set of all tasks. Each job $j \in J$ is a vector $\left(t_{1}, t_{2}, t_{3}\right)$ of three tasks, one at each stage (the first component is the task in the first stage, etc.). Set $T$ can be partitioned as follows: $T=T^{1} \cup T^{2} \cup T^{3}$, with $T^{i}$ the set of all tasks of stage $i(i=1,2,3)$. A second partition is $T=T_{C} \cup T_{U} \cup T_{L}$, where the set $T_{C}$ contains all activities related to trailers that will remain coupled to the truck, $T_{U}$ gathers all tasks related to an uncoupled trailer that has to be unloaded, and $T_{L}$ contains all tasks pertaining to an uncoupled trailer that has to be loaded. Each task $t \in T^{1}$ has a ready time $r_{t}$; for $t \in T_{L}, r_{t}=0$. With each task 
Table 1: Data for the example instance. Type 'C' are coupled jobs, type ' $U$ ' are unload jobs and type ' $\mathrm{L}$ ' are load jobs. All parameters (weight, ready time,....) pertain to the appropriate tasks of each job.

\begin{tabular}{llllll}
\hline job & weight & $\begin{array}{l}\text { ready } \\
\text { time }\end{array}$ & $\begin{array}{l}\text { processing } \\
\text { time }\end{array}$ & $\begin{array}{l}\text { due } \\
\text { date }\end{array}$ & type \\
\hline 1 & 2 & 2 & 12 & 17 & $\mathrm{C}$ \\
2 & 3 & 3 & 12 & & $\mathrm{U}$ \\
3 & 3 & 1 & 14 & & $\mathrm{U}$ \\
4 & 2 & 0 & 10 & 15 & $\mathrm{~L}$ \\
5 & 1 & 0 & 11 & 25 & $\mathrm{~L}$ \\
\hline
\end{tabular}

$t \in T^{2}$ we associate a processing time $p_{t}$, denoting the time to load or unload the trailer. Each third-stage loading task $t \in T_{L} \cap T^{3}$ has a due date $d_{t}$, which is based on the agreed arrival time at the customer. Each third-stage coupled task $t \in T_{C} \cap T^{3}$ also has a due date, which creates a time window for the coupled trailers. This window is meant to restrict the trucker's time at the site. Each of the tasks $t \in\left(T_{U} \cap T^{2}\right) \cup\left(T_{L} \cap T^{3}\right) \cup\left(T_{C} \cap T^{3}\right)$ also has a weight $w_{t}$, representing the importance of the job. The resources in the second stage are $m<n$ identical docks, and $\tau<m$ identical terminal tractors execute both the first and the third stage of the uncoupled trailers. The trucks that transport coupled trailers are not explicitly modeled as resources as they are not shared among the different trailers. Each machine (either a dock or a tractor) can process at most one task at a time and preemption of a task is not possible.

During the transportation stages one and three, the dock is also considered to be occupied, mainly for safety reasons. As a consequence, a stage-two activity always starts immediately at the end of the corresponding stage-one activity; there is no capacity issue. A stagethree activity of a coupled trailer will also start immediately. A stage-three activity of an uncoupled trailer, on the other hand, can start as soon as the corresponding stage-two activity is finished, but will regularly be delayed because of unavailability of tractors. This leads to the phenomenon of blocking: as long as the stage-three activity for a trailer is not executed, the assigned dock remains occupied although stage two may already be completed. Consequently, also the 'dock'-resources are not exclusively tied to only one stage. An example instance with five trailers $(n=5)$ is described in Table 1. A feasible schedule with one tractor $(\tau=1)$ and three docks $(m=3)$ is depicted in Figure 1. Each job is visually represented by three blocks, one for each stage. White blocks represent occupation of the dock and black blocks represent occupation of both the tractor and the dock. Note that the tractor is not assigned to the transportation of job one, since it is a coupled job. The hatched block represents blocking: trailer three remains at dock two although unloading is already finished because the terminal tractor is occupied at another dock during time period 17 .

Informally, our goal is to have all outgoing and coupled trailers ready for transport by their due date and also to perform all tasks as quickly as possible. The first objective of respecting due dates is far more important than the overall desire of 'early' processing, and 


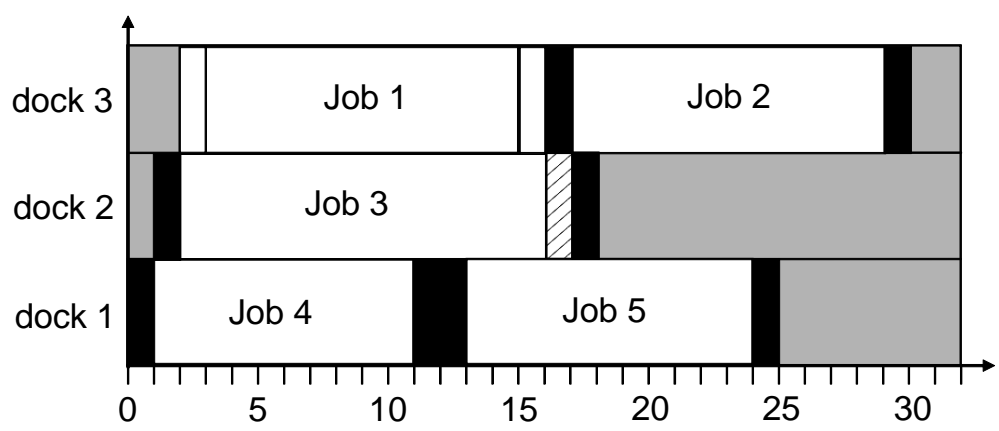

Figure 1: A feasible schedule for $m=3$ and $\tau=1$.

we opt for lexicographic optimization. The primary objective is to live up to the due dates as well as possible. The minimization of the weighted sum of completion times is our secondary objective, where for incoming jobs the completion time of stage two is important, while for coupled and outgoing jobs we focus on the completion time of stage three. In Berghman et al. (to appear), optimization only of the second objective was studied, while due-date violation was forbidden (due dates were deadlines). In practice, however, it turns out that the due dates are tight and a feasible plan without violation might not exist. After discussions with the site management, we have opted for modeling the primary objective by means of two components: we minimize the weighted sum of the number of late coupled and outgoing trailers and the tardiness of those trailers. Formally, our objectives are

$$
\min z_{1}=\sum_{\substack{t \in\left(T_{C} \cap T^{3}\right) \\ \cup\left(T_{L} \cap T^{3}\right)}} \theta_{t}+\alpha \delta_{t}
$$

and

$$
\min z_{2}=\sum_{\substack{t \in\left(T_{C} \cap T^{3}\right) \\ \cup\left(T_{L} \cap T^{3}\right) \\ \cup\left(T_{U} \cap T^{2}\right)}} w_{t} C_{t},
$$

where $C_{t}$ is the completion time of task $t, \theta_{t}=\max \left\{C_{t}-d_{t} ; 0\right\}$ is the tardiness of $t$ and $\delta_{t}$ is a binary indicator equal to 1 if $\theta_{t}>0$, and 0 otherwise.

The composite objective $z_{1}$ was chosen because lower tardiness is better for a trailer, but we may prefer having one trailer late by two time periods over having two trailers late each by one time period, because each tardiness occurrence will give rise to communication with the client, loss of time and a possible loss in revenues and/or reputation. For this reason, we also incorporate the number of trailers late. The value $\alpha \geq 0$ serves as a scaling parameter between these two client-oriented performance measures. The weighted completion-time objective $z_{2}$ is also convenient for our setting: all the incoming goods will be stored in the warehouse as early as possible and all outgoing trailers will be in the parking zone as quickly as possible, ready for transportation towards the client. Additionally, in case of coupled trailers, we also reduce the trucker's stay on site. Optimization of $z_{1}$ has priority over $z_{2}$ : improving $z_{1}$ is crucial, even if it causes a worsening in $z_{2}$ (hierarchical optimization). 


\section{Integer programming}

Various IP formulations were explored in Berghman et al. (to appear) for minimizing $z_{2}$ with deadlines. A time-indexed formulation was consistently found to be the most efficient. For this reason, we will adapt that formulation to our multicriteria setting with due dates. Let (time) period $u$ be the time interval $[u-1, u[$. For all tasks $t \in T$ and for all time periods $u \in H_{t}$, we define variable

$$
x_{t u}= \begin{cases}1 & \text { if task } t \text { starts in period } u, \\ 0 & \text { otherwise }\end{cases}
$$

with $H_{t}$ the time window for $t \in T$. Specifically, we choose $H_{t}=\left\{r_{t}+1, \ldots, H_{\max }-p_{t}-1\right\}$ if $t \in T^{1}, H_{t}=\left\{r_{t}+2, \ldots, H_{\max }-p_{t}\right\}$ if $t \in T^{2}$ and $H_{t}=\left\{r_{t}+2+p_{t}, \ldots, H_{\max }\right\}$ if $t \in T^{3}$, where $H_{\text {max }}$ is the length of the time horizon. Furthermore, for all tasks $t \in\left(T_{C} \cap T^{3}\right) \cup\left(T_{L} \cap T^{3}\right)$, consider $\theta_{t}$ and $\delta_{t}$ as defined in Section 3. A linear formulation with these variables and with two objectives is:

$$
\begin{gathered}
\min z_{1}=\sum_{\substack{t \in\left(T_{C} \cap T^{3}\right) \\
\cup\left(T_{L} \cap T^{3}\right)}} \theta_{t}+\alpha \delta_{t} \\
\min z_{2}=\sum_{t \in T_{U} \cap T^{2}} w_{t}\left(\left(\sum_{u \in H_{t}} u x_{t u}\right)-1+p_{t}\right)+\sum_{\substack{t \in\left(T_{C} \cap T^{3}\right) \\
\cup\left(T_{L} \cap T^{3}\right)}} w_{t}\left(\sum_{u \in H_{t}} u x_{t u}\right)
\end{gathered}
$$

subject to

$$
\begin{array}{cr}
\sum_{u \in H_{t}} x_{t u}=1 & \forall t \in T \\
\sum_{u \in H_{t}} u x_{t u}-d_{t}-\delta_{t} H_{\max } \leq 0 & \forall t \in\left(T_{C} \cap T^{3}\right) \cup\left(T_{L} \cap T^{3}\right) \\
\sum_{u \in H_{t}} u x_{t u}-d_{t}-\theta_{t} \leq 0 & \forall t \in\left(T_{C} \cap T^{3}\right) \cup\left(T_{L} \cap T^{3}\right) \\
\sum_{\left(t_{1}, t_{2}, t_{3}\right) \in J}\left(x_{t_{1} u}+x_{t_{3} u}+\sum_{v \leq u}\left(x_{t_{2} v}-x_{t_{3} v}\right)\right) \leq m & \forall u \in\left\{1, \ldots, H_{\max }\right\} \\
\sum_{\left(t_{1}, t_{2}, t_{3}\right) \in J_{U} \cap J_{L}}\left(x_{t_{1} u}+x_{t_{3} u}\right) \leq \tau & \forall u \in\left\{1, \ldots, H_{\max }\right\} \\
x_{t_{1} u}-x_{t_{2}, u+1}=0 & \forall\left(t_{1}, t_{2}, t_{3}\right) \in J ; \forall u \in H_{t_{1}} \\
\sum_{v=1}^{u} x_{t_{3} v}-\sum_{v=1}^{u} x_{t_{2} v} \leq 0 & \forall\left(t_{1}, t_{2}, t_{3}\right) \in J ; \forall \in\left\{1, \ldots, H_{\max }\right\} \\
x_{t u} \in\{0,1\} & \forall t \in T ; \forall u \in H_{t} \\
\delta_{t} \in\{0,1\} & \forall t \in\left(T_{C} \cap T^{3}\right) \cup\left(T_{L} \cap T^{3}\right) \\
\theta_{t} \geq 0 & \forall t \in\left(T_{C} \cap T^{3}\right) \cup\left(T_{L} \cap T^{3}\right)
\end{array}
$$


Objective function (1) minimizes the weighted sum of the number of late coupled and outgoing trailers and the tardiness of those trailers and objective (2) minimizes the weighted completion time of the stage-two activities of the incoming trailers and the stage-three activities of the coupled and outgoing trailers. Constraint (3) requires each task to be processed exactly once. Constraint (4) establishes whether a job is late or not and constraint (5) measures the tardiness. Constraint (6) ensures that in each time period, at most $m$ docks are occupied. Constraint (7) enforces the capacity of the terminal tractors. Constraints (8) and (9) implement the precedence constraints between the three stages. We observe that a stage-two task can always begin immediately after the corresponding stage-one task has been completed. For reasons of clarity, the model above includes all variables relating to the three stages, but for actual computations the stage-two variables are eliminated via substitution according to (8). Optimization proceeds in two steps: first, objective (1) is optimized subject to constraints (3)-(12), leading to objective value $z^{*}$. Subsequently, the constraint $z_{1} \leq z^{*}$ is added and then objective (2) is optimized. The obtained objective value will be denoted as $z_{2}\left(z^{*}\right)$.

All algorithms in this article were encoded in $\mathrm{C}$ using the Microsoft Visual Studio programming environment, and executed on a Dell Latitude D630 with an Intel Pentium-4 2.2-GHz processor and 2 GB RAM, equipped with Windows 7. CPLEX version 10.2 is used to solve the IP and LP models. The length of the planning horizon $H_{\max }=120$, representing a working day in which each time unit corresponds to 10 minutes. Based on Sadykov and Wolsey (2006) and Berghman et al. (to appear), instances were created in the following way. In line with the current situation in the case studied, $25 \%$ of the trailers remains coupled, $45 \%$ is uncoupled and has to be loaded, the remaining $30 \%$ are uncoupled trailers to be loaded. The ready times for the coupled and the incoming trailers are integers randomly selected out of $[0,64]$ and the weights of all trailers are randomly selected out of $\{1,2,3\}$ (each value has equal probability). The processing times $p_{t}=1+X$ with $X$ binomially distributed with 16 trials and a probability of 0.5. The due dates for the coupled trailers are obtained as $d_{t}=r_{t}+p_{t}+18$, while the due dates for the outgoing jobs are calculated in the following way: $d_{j}=\max \left\{d_{j}^{\prime}, r_{j}+\max _{k \in J}\left\{p_{k}\right\}\right\}$ with $d_{j}^{\prime} \in[\beta-10, \beta+10]$ and $\beta=\frac{\sum_{j \in J} p_{j} * 0.5}{m}$. Table 2 displays the objective values and the computation times for medium-sized instances for both the IP formulations and for their LP relaxations; the latter yield lower bounds $L B_{1}$ on $z_{1}$ and $L B_{2}\left(z_{1}\right)$ on $z_{2}\left(z_{1}\right)$. We report these bounds because they may be useful in later sections. In all implementations, we choose $\alpha=1$ unless otherwise mentioned. Here and below, 'time ${ }_{i}$ ' represents the time spent by the relevant procedure on optimizing objective $z_{i}$. On average, $L B_{1}$ requires less computation time than $L B_{2}$, but the bound is not tight. $L B_{2}$, on the other hand, is usually a rather tight bound. The computation times for the LP relaxation are sometimes larger than those for the IP, which might be caused by the fact that CPLEX performs considerable preprocessing (ILOG, 2008).

For larger and more realistic instances with more trailers per dock (up to 48 gates, 480 trailers and over 1000 operations), CPLEX is no longer able to produce guaranteed optimal solutions: see Table 3. In some cases, CPLEX is even unable to find a feasible solution within one hour of computation time, or is aborted because of memory problems. 
Table 2: Computational results for medium-sized instances for the IP formulations and the LP relaxations.

\begin{tabular}{|lll|rrrr|rrrr|}
\hline \multirow{2}{*}{$m$} & $n$ & $\tau$ & \multicolumn{7}{|c|}{ IP } & \multicolumn{3}{c|}{ LP } \\
& & & $z_{1}$ & $z_{2}\left(z_{1}\right)$ & time $_{1}(\mathrm{~s})$ & time $_{2}(\mathrm{~s})$ & $L B_{1}$ & $L B_{2}\left(z_{1}\right)$ & time $_{1}(\mathrm{~s})$ & time $_{2}(\mathrm{~s})$ \\
\hline 20 & 80 & 2 & 190 & 6167 & 128.46 & 163.66 & 158.31 & 5884.01 & 63.93 & 59.46 \\
20 & 80 & 3 & 36 & 5694 & 74.39 & 88.90 & 2.84 & 5622.54 & 35.79 & 46.66 \\
20 & 90 & 2 & 227 & 6570 & 142.66 & 303.38 & 187.55 & 6218.63 & 151.68 & 148.66 \\
20 & 90 & 3 & 45 & 5883 & 83.97 & 78.18 & 0.00 & 5672.36 & 28.01 & 74.69 \\
20 & 100 & 2 & 256 & 8187 & 302.37 & 300.89 & 206.20 & 7780.08 & 204.01 & 530.29 \\
20 & 100 & 3 & 10 & 7233 & 86.83 & 70.80 & 0.00 & 6960.89 & 34.38 & 100.91 \\
\hline 24 & 96 & 2 & 830 & 7815 & 128.66 & 196.84 & 785.49 & 7494.80 & 90.33 & 165.29 \\
24 & 96 & 3 & 722 & 6704 & 57.52 & 105.40 & 711.88 & 6611.82 & 21.84 & 47.62 \\
24 & 108 & 2 & 464 & 9953 & 211.63 & 338.17 & 425.01 & 9437.84 & 195.90 & 244.35 \\
24 & 108 & 3 & 98 & 8414 & 155.95 & 154.61 & 21.87 & 7954.48 & 168.42 & 104.39 \\
24 & 120 & 2 & 522 & 11371 & 593.42 & 457.97 & 482.99 & 10828.57 & 276.23 & 402.25 \\
24 & 120 & 3 & 73 & 9119 & 187.38 & 357.40 & 0.00 & 8791.87 & 75.49 & 146.93 \\
\hline 28 & 112 & 2 & 660 & 8987 & 816.62 & 324.84 & 629.70 & 8728.63 & 222.35 & 282.79 \\
28 & 112 & 3 & 257 & 7470 & 273.87 & 611.65 & 212.76 & 7202.65 & 197.23 & 148.53 \\
28 & 126 & 2 & 719 & 12492 & 685.43 & 656.40 & 679.11 & 12024.77 & 249.03 & 378.43 \\
28 & 126 & 3 & 219 & 10138 & 682.67 & 269.51 & 152.26 & 9792.58 & 197.26 & 213.42 \\
28 & 140 & 2 & 894 & 14302 & 1353.81 & 1066.44 & 832.88 & 13515.94 & 364.31 & 769.36 \\
28 & 140 & 3 & 262 & 11436 & 276.45 & 580.84 & 165.87 & 10664.83 & 370.41 & 629.12 \\
\hline 32 & 128 & 2 & 450 & 9792 & 680.76 & 386.35 & 410.39 & 9339.38 & 179.74 & 240.24 \\
32 & 128 & 3 & 202 & 8806 & 188.81 & 185.47 & 140.16 & 8550.32 & 121.70 & 90.72 \\
32 & 144 & 2 & 383 & 11942 & 1092.57 & 638.58 & 318.13 & 11255.33 & 310.01 & 501.04 \\
32 & 144 & 3 & 79 & 10715 & 226.43 & 192.76 & 0.00 & 10113.47 & 60.69 & 228.65 \\
32 & 160 & 2 & 578 & 13378 & 1380.77 & 897.79 & 531.90 & 12741.88 & 369.92 & 667.24 \\
32 & 160 & 3 & 193 & 11574 & 450.80 & 484.42 & 96.30 & 11102.04 & 348.67 & 272.47 \\
\hline
\end{tabular}

\section{Schedule representation and generation scheme}

It turns out (based on the previous section as well as on preliminary results for the exact B\&B algorithm proposed in Section 6) that we cannot solve realistic instances to guaranteed optimality within reasonable running times and in the remainder of this text, we therefore resort to the development of heuristic procedures for solving the dock assignment problem. The procedures will be discussed in the following sections and afterwards compared experimentally. In this section, we first explain the schedule representation and the schedule generation scheme that will be used by those procedures.

Similar to most improvement heuristics for scheduling problems, we will not operate directly on a schedule but rather on a representation of a schedule that admits an efficient and effective functioning of the algorithm. We opt for an (ordered) activity list, which will also be referred to as a 'sequence' or 'permutation'; similar choices have been made in a number of branching algorithms (see, e.g., Baker, 1974; Azizoğlu and Kirca, 1999). The activities of stage two are not included in the list because they always start immediately after the corresponding stage-one activity. The third-stage activities of the coupled trailers are not included either since they start immediately after the corresponding stage-two activity. As 
Table 3: Computational results for large instances for the IP formulations and the LP relaxations. For some instances, CPLEX does not find any feasible solution within one hour ('no feas sol') or encounters memory problems. The procedure is interrupted after one hour of running time; such cases are written as ' $>1$ h' and the IP value $z_{2}\left(z_{1}\right)$ may then not be optimal. When also $z_{1}$ is not optimized to completion, an asterisk ' $*$ ' is included. A double asterisk ${ }^{(* *}$ ' indicates that CPLEX is unable to optimize the secondary objective for lack of an integer solution (which is needed to provide a primary objective value).

\begin{tabular}{|c|c|c|c|c|c|c|c|c|c|}
\hline \multirow{2}{*}{$m$} & \multirow{2}{*}{$n$} & \multirow{2}{*}{$\tau$} & \multicolumn{3}{|c|}{ IP } & \multicolumn{4}{|c|}{ LP } \\
\hline & & & $z_{1}$ & $z_{2}\left(z_{1}\right)$ & time $(\mathrm{s})$ & $L B_{1}$ & $L B_{2}\left(z_{1}\right)$ & time $_{1}(\mathrm{~s})$ & $\operatorname{time}_{2}(\mathrm{~s})$ \\
\hline 36 & 216 & 4 & 489 & 19775 & 2555.483 & 312.58 & 18705.58 & 952.12 & 757.14 \\
\hline 36 & 216 & 5 & $119^{*}$ & 22045 & $>1 \mathrm{~h}$ & 0.00 & 17416.76 & 169.79 & 780.82 \\
\hline 36 & 288 & 5 & $412^{*}$ & 31163 & $>1 \mathrm{~h}$ & 0.00 & 26244.73 & 340.02 & 3086.55 \\
\hline 36 & 288 & 6 & no fe & sol & & 0.00 & $* *$ & 228.56 & $* *$ \\
\hline 36 & 360 & 6 & no fe & sol & & 0.00 & ** & 628.78 & $* *$ \\
\hline 36 & 360 & 7 & no fe & sol & & 0.00 & $* *$ & 536.89 & $* *$ \\
\hline 40 & 240 & 4 & 800 & 21584 & 2792.29 & 618.11 & 20267.06 & 839.08 & 1916.60 \\
\hline 40 & 240 & 5 & 298 & 19275 & 2322.02 & 30.25 & 18173.69 & 357.43 & 588.00 \\
\hline 40 & 320 & 5 & 522 & 36205 & $>1 \mathrm{~h}$ & 0.00 & 30582.60 & 199.00 & 30582.60 \\
\hline 40 & 320 & 6 & $199^{*}$ & 35310 & $>1 \mathrm{~h}$ & 0.00 & 30044.87 & 142.72 & 1776.25 \\
\hline 40 & 400 & 6 & no fe & sol & & 0.00 & $* *$ & 896.01 & $* *$ \\
\hline 40 & 400 & 7 & no fe & sol & & 0.00 & $* *$ & 717.54 & $* *$ \\
\hline 44 & 264 & 5 & $542^{*}$ & 27026 & $>1 \mathrm{~h}$ & 363.68 & 21452.53 & 2132.47 & 1077.16 \\
\hline 44 & 264 & 6 & $190^{*}$ & 25841 & $>1 \mathrm{~h}$ & 0.00 & 20218.41 & 73.24 & 574.41 \\
\hline 44 & 352 & 6 & no fe & sol & & 0.00 & $* *$ & 194.47 & ** \\
\hline 44 & 352 & 7 & 341 & 37617 & $>1 \mathrm{~h}$ & 0.00 & 32515.95 & 157.88 & 1763.71 \\
\hline 44 & 440 & 7 & mem & y probl & & 0.00 & $* *$ & 569.42 & ** \\
\hline 44 & 440 & 8 & no fe & sol & & 0.00 & $* *$ & 1048.15 & $* *$ \\
\hline 48 & 288 & 5 & 816 & 30831 & $>1 \mathrm{~h}$ & 585.84 & 24887.89 & 1911.34 & 1671.83 \\
\hline 48 & 288 & 6 & 346 & 24099 & 2650.91 & 18.15 & 22871.72 & 811.27 & 927.27 \\
\hline 48 & 384 & 6 & mem & y probl & & 500.13 & $* *$ & 574.03 & ** \\
\hline 48 & 384 & 7 & mem & y probl & & 500.13 & $* *$ & 563.79 & $* *$ \\
\hline 48 & 480 & 7 & mem & y probl & & 0.00 & $* *$ & 1548.21 & $* *$ \\
\hline 48 & 480 & 8 & mem & y probl & & 0.00 & $* *$ & 1203.81 & $* *$ \\
\hline
\end{tabular}

a consequence, the length of the permutation will be $2 n-c$ with $c$ the number of coupled trailers. A schedule representation is transformed into a schedule by means of a schedule generation scheme (for details, see Kolisch, 1996). We implement a so-called serial generation scheme, which iteratively selects the next task in the list and schedules it as early as possible, taking ready times and capacity constraints into account. Only sequences that respect the intra-job precedence constraints will be considered: for each uncoupled trailer, the stage-one task has to precede the stage-three task. The sequence $\left(4,3,1,2,4^{\prime}, 5,3^{\prime}, 5^{\prime}, 2^{\prime}\right)$, for instance, is transformed into to the schedule in Figure 1, with a number without prime representing a stage-one activity and a number with prime a stage-three activity. Note that this is not the only sequence leading to this schedule. It can be shown (e.g. Kolisch, 1996) that with a regular objective function, which is the case both for $z_{1}$ and for $z_{2}$, at least one sequence is mapped to an optimal schedule by the serial generation scheme.

The blocking phenomenon may hamper a straightforward transformation of an activity 


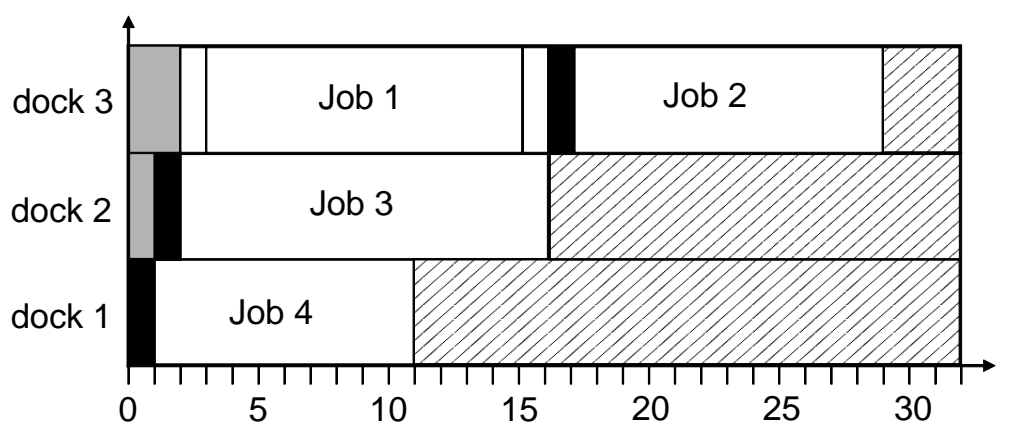

Figure 2: The partial schedule for the permutation $\left(4,3,1,2,5,4^{\prime}, 3^{\prime}, 5^{\prime}, 2^{\prime}\right)$, after which the generation scheme breaks down.

list into a feasible schedule. An illustration is provided in Figure 2, where all docks are blocked after the stage-one activities of trailers 4,3 and 2 are scheduled because the corresponding stage-three activities are not yet scheduled. The next task in the permutation is the stage-one activity of trailer 5 , for which there is no free dock.

We call a permutation valid if the generation scheme finds a free dock at each iteration in which a stage-one activity is planned, so that the capacity constraints are always respected. Consequently, a valid permutation generates a feasible schedule, since all permutations considered respect the inter-stage precedences. Define $\nu(t)$ as the number of stage-one activities related to an uncoupled trailer minus the number of stage-three activities from the start of the permutation up to, but not including, position $t(t \in\{2, \ldots, 2 n-c\})$.

Lemma 1 A permutation is valid if and only if for each position $t \in\{2,2, \ldots, 2 n-c\}$ filled with a stage-one activity, $\nu(t)<m$.

Proof: When the generation scheme reaches position $t$ in permutation, the number of free docks is exactly $m-\nu(t)$.

In the example provided in Figure $2, \nu(5)=3=m$, so the permutation is not valid.

For a given permutation $L$, we define a new permutation $V(L)$ obtained by traversing the permutation from left to right and monitoring $\nu(t)$ for each position $t$. Each time when $\nu(t)=m$, the first stage-three task in the list after position $t$ with stage-one task before position $t$ is inserted at position $t$ and the tasks in between are shifted one position to the right.

Lemma 2 For any list $L$, the list $V(L)$ is valid.

Proof: Straightforward, from Lemma 1.

As an illustration, the invalid (i.e., not valid) permutation $\left(4,3,1,2,5,4^{\prime}, 3^{\prime}, 5^{\prime}, 2^{\prime}\right)$ can be transformed into the valid permutation $\left(4,3,1,2,4^{\prime}, 5,3^{\prime}, 5^{\prime}, 2^{\prime}\right)$ using the above strategy. The resulting schedule was given in Figure 1. 


\section{Branch and bound}

The IP formulation presented in Section 4 is frequently unable to produce any feasible solution within the allotted runtime (see Table 3). In this section, we describe a B\&B algorithm for the dock assignment problem. Its running times for obtaining guaranteed optimal solutions have turned out to be excessive for larger instances, regularly even longer than for the IP formulation of Section 4, and we will therefore examine the performance of the algorithm especially in a truncated mode (interrupted after a predetermined time period); the algorithm will be referred to as 'truncated B\&B'. An alternative way of exploring the enumeration tree only partially via beam search will be studied in Section 7 .

Below, we first comment the branching strategy (Section 6.1) and subsequently provide more details on the bounding procedures (6.2) and on parameterization (6.3).

\subsection{Branching strategy}

The second stage of the dock assignment problem corresponds to a parallel-machine scheduling problem. For minimizing the total weighted completion time on parallel machines without ready times, it is a dominant decision to sequence the jobs allocated to a given machine by non-decreasing weighted processing time. Therefore, optimization routines need only be concerned with establishing appropriate job-machine assignments. Azizoğlu and Kirca (1999), for example, propose a B\&B algorithm for minimization of the total weighted completion time on parallel machines where at each level of the enumeration tree, a given job is assigned to one of the machines. Procedures for identical parallel-machine problems with ready times (see, e.g., Nessah et al., 2008) or which minimize the (weighted) tardiness (see, e.g., Azizoğlu and Kirca, 1998; Shim and Kim, 2007) rely on the fact that an optimal schedule can be constructed by assigning jobs to earliest available machines one by one according to an optimal job priority list (Baker, 1974). Therefore, enumeration schemes fix the elements of a priority list from first to last such that a subproblem corresponds to a partial schedule: a node at the $i^{\text {th }}$ level of the tree represents a partial schedule in which the first $i$ positions have been filled and branching from a node consists in appending an unscheduled job to the end of the partial list.

For the three-stage scheduling problem studied in this article, we will also enumerate priority lists (permutations) by selecting the tasks in the list from the first to the last. A subproblem corresponding to a node at depth $l$ of the search tree is to determine the last $(2 n-c-l)$ elements of the permutation, and branching at this node is performed by fixing the $(l+1)^{t h}$ task in the list; the already sequenced tasks are called fixed tasks. Nodes for which the fixed tasks do not respect the intra-job precedences or the capacity constraints are immediately discarded. Each level of the tree constitutes a partition of all valid permutations, in which each node represents a subset of permutations with the same initial elements. From this subset, we choose one particular permutation that will be called the representative of the node, which is the permutation that most closely resembles the representative of the parent node and respects the branching decision. We refer to the corresponding objective values by $r e p_{1}$ and $r e p_{2}$. 


\subsection{Bounds}

A feasible solution to constraints (3)-(12) yields an upper bound $U B_{1}$ to $z_{1}$ and also an upper bound $U B_{2}\left(U B_{1}\right)$ to $z_{2}$ that is conditional on an upper-bound constraint $z_{1} \leq U B_{1}$. For the example of Table 1, the schedule represented by Figure 1 gives an upper bound $U B_{1}=0$ to $z_{1}$ and an upper bound $U B_{2}(0)=216$ to $z_{2}$. Upper bounds are global bounds: they hold for all nodes in the search tree. Obviously, a value $U B_{2}(z)$ can also serve as $U B_{2}\left(z^{+}\right)$with $z<z^{+}$. A lower bound $L B_{1}$ for $z_{1}$ is obtained as the optimal solution for a relaxation; a lower bound $L B_{2}\left(U B_{1}\right)$ for $z_{2}$ corresponds to the optimal solution for a relaxed problem with the addition of an upper-bound constraint $z_{1} \leq U B_{1}$. Lower bounds are local bounds: they are specific to one node in the search tree and all its children; a lower bound in the root node is a global bound.

\subsubsection{General}

In each node, the partial schedule with the fixed tasks is monitored and a relaxation is solved of the scheduling problem of the remaining tasks, with capacities varying over the time periods (so in the IP formulation, for instance, the right-hand side of (6) and (7) can be different for different $u$ ). A lower bound is then the sum of the exact objective value for the fixed tasks and a bound for the contribution of the other tasks. It holds that $L B_{2}(z) \geq L B_{2}\left(z^{+}\right)$if $z^{+}>z$, since the optimization problem for $z^{+}$is a relaxation of the problem associated with $z$.

When for a certain node $L B_{1} \geq U B_{1}$, the node will be pruned. The same holds for a node where $L B_{1}=U B_{1}$ and $L B_{2}\left(U B_{1}\right) \geq U B_{2}\left(U B_{1}\right)$. In this way, a node is eligible for further exploration only when $L B_{1}<U B_{1}$ or when $L B_{1}=U B_{1}$ and $L B_{2}\left(U B_{1}\right)<U B_{2}\left(U B_{1}\right)$.

Table 3 shows that CPLEX needs excessive runtimes for solving the LP relaxation; a similar problem arises for the other formulations of Berghman et al. (to appear). Two different relaxations therefore are considered for producing lower bounds. The first one is Lagrangian relaxation; this method is explained in more detail in Section 6.2.2. The second option is a relaxation of the number of tractors: if we set $\tau=\infty$ then a parallel-machine scheduling problem results in which only stage two needs to be considered, if the processing times are extended by the transportation times. The resulting bound can be computed by a timeindexed parallel-machine formulation based on the one of Berghman et al. (to appear). The tractors are the bottleneck machines in our configuration and it turns out that $L B_{1}^{P M}=0$ for all tested instances, so it is possible to meet all due dates in the relaxation. Consequently, only the corresponding $z_{2}$-bound $L B_{2}^{P M}\left(U B_{1}\right)$ will be used for pruning. For each such relaxation we can also produce a permutation, by sequencing the jobs in non-decreasing order of their starting times and then replacing each coupled job by its corresponding stage-one task and each uncoupled job by its corresponding stage-one and stage-three task. The resulting feasible schedule yields an upper bound. The relaxed solutions found by Lagrangian relaxation (Section 6.2.2) can similarly be transformed into upper bounds by ordering all relevant tasks by their starting time. The idea of transforming a solution to a relaxation into a feasible solution is not very common for 'standard' B\&B algorithms, but has been suggested already in a context of Lagrangian relaxation (see Möhring et al. 1999, 2003). 


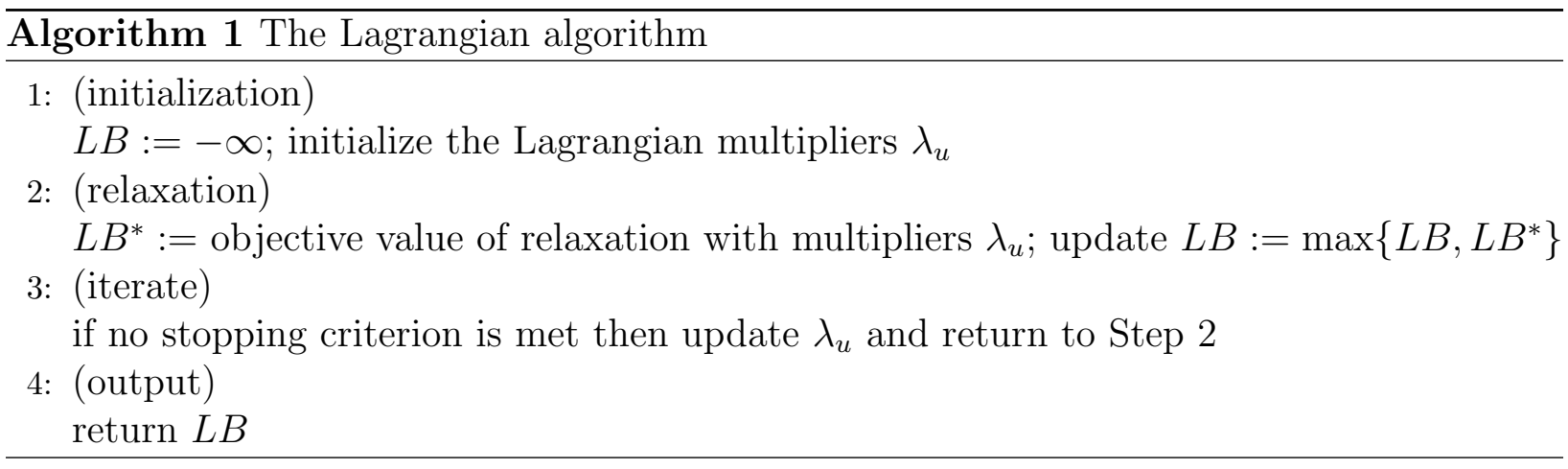

\subsubsection{Lagrangian relaxation}

To calculate $L B_{1}^{L R}$, the capacity constraints of both resource types (docks and tractors) are relaxed by means of Lagrange multipliers (see, e.g., Fisher, 1981). Additionally, for computing $L B_{2}^{L R}\left(U B_{1}\right)$, the extra constraint $z_{1} \leq U B_{1}$ is also relaxed. In this way, easily solvable independent job-level subproblems are obtained where the multipliers act as prices that regulate the use of the machines. For each task, the optimal starting time strikes a balance between these machine prices and the original objective function, either the number of jobs late and the total tardiness or the weighted completion time. The relaxed problem is solved multiple times, and at each iteration the multipliers are updated by means of subgradient optimization. An overview of the complete procedure is given as Algorithm 1. The two stopping criteria consist of an upper limit on the number of iterations and on the running time; in our implementations, these limits are 60 seconds and 1000 iterations in the root node, and 5 seconds and 10 iterations for all other search nodes. When the optimization is halted, a relaxed solution is obtained, which may or may not be feasible. The two most important steps in the computation are explained next.

Relaxation The subproblem per job entails the choice of starting times for the activities so as to minimize the objective value subject to the intra-job precedence constraints. For the uncoupled trailers, only the tasks of stages one and three need to be scheduled explicitly since the stage-two task is started immediately after stage one. We evaluate each precedence-feasible combination of starting times for stages one and three and select one with the lowest cost. For the coupled trailers, it suffices to schedule only stage one explicitly and select a starting time with lowest cost. A natural implementation has a running time that is linear in the number of jobs and quadratic in the length of the planning horizon.

Updating the Lagrangian multipliers Subgradient optimization is an iterative procedure that generates new multipliers starting from an initial set in a systematic fashion. This procedure attempts to find values for the multipliers that yield the maximum lower bound. The initial multipliers are of the form $\lambda_{u 0}^{\prime}=\beta \frac{H_{\max }}{m}$ for the docks and $\lambda_{u 0}^{\prime \prime}$ $=\beta \frac{H_{\max }}{\tau}$ for the tractors $\left(u \in\left\{1, \ldots, H_{\max }\right\}\right)$. The subgradients for the relaxed constraints are $S G_{u}^{\prime}=\sum_{\left(t_{1}, t_{2}, t_{3}\right) \in J}\left(x_{t_{1} u}+x_{t_{3} u}+\sum_{v \leq u}\left(x_{t_{2} v}-x_{t_{3} v}\right)\right)-m_{u}$ for the docks and $S G_{u}^{\prime \prime}=\sum_{\left(t_{1}, t_{2}, t_{3}\right) \in J_{U} \cup J_{L}}\left(x_{t_{1} u}+x_{t_{3} u}\right)-\tau_{u}$ for the tractors, where $m_{u}$ represents the number of available docks during time period $u$ and $\tau_{u}$ is the number of available 
Table 4: Comparison of different settings for the B\&B procedure.

\begin{tabular}{|l|l||ll|}
\hline & Setting & $z_{1}$ & $z_{2}\left(z_{1}\right)$ \\
\hline \multirow{5}{*}{ Branching strategy } & (1) $L B_{1}^{L R}$ and $L B_{2}^{L R}$ & $10.03 \%$ & $1.03 \%$ \\
& $(2) L B_{1}^{L R}$ and $L B_{2}^{P M}$ & $42.09 \%$ & $-2.12 \%$ \\
& $(3) L B_{1}^{L R}$ and $U B_{2}$ & $0.00 \%$ & $0.00 \%$ \\
& $(4) U B_{1}$ and $L B_{2}^{L R}$ & $20.72 \%$ & $-3.65 \%$ \\
\hline \multirow{3}{*}{ Initial solution } & $(1) L B_{1}^{L R}$ & $0.00 \%$ & $0.00 \%$ \\
& $(2) L B_{2}^{L R}$ & $9.59 \%$ & $-0.56 \%$ \\
& $(3) L B_{2}^{P M}$ & $24.45 \%$ & $0.68 \%$ \\
\hline
\end{tabular}

tractors during $u$. The step size is $w^{-1}$, with $w$ increasing by $X$ every $Y$ iterations. The multipliers are updated as follows in iteration $i: \lambda_{u i}^{\prime}=\max \left\{0, \lambda_{u, i-1}^{\prime}+w^{-1} S G_{u}^{\prime}\right\}$ for the docks and $\lambda_{u i}^{\prime \prime}=\max \left\{0, \lambda_{u, i-1}^{\prime \prime}+w^{-1} S G_{u}^{\prime \prime}\right\}$ for the tractors $(i=1,2, \ldots)$. The multipliers for the additional constraint on $z_{1}$ in the optimization of $z_{2}$ are updated in a similar fashion. Some small experiments were run for parameterization.

\subsection{Parameterization}

Table 4 shows the results of an experiment run on four large instances ${ }^{1}$ in order to evaluate the most convenient algorithmic choices for the truncated $\mathrm{B} \& \mathrm{~B}$ algorithm. We report the gap of the primary and secondary objective compared to one 'base' setting (corresponding to $0.00 \%$ in the table), calculated as the relative difference in objective value. Each setting is interrupted after 20 minutes. Four different branching strategies have been implemented. Each strategy uses two 'estimates' for the best value of one objective achievable in each node; each estimate is either an upper or a lower bound. The first value is used to decide which node to explore next, and a second bound serves as tie breaker. Superscript ' $L R$ ' refers to Lagrangian relaxation, ' $P M$ ' stands for parallel-machine relaxation. In strategies (1) and (2), the upper bound $U B_{1}$ on $z_{1}$ imposed for computing $L B_{2}\left(U B_{1}\right)$ is the minimum of $r e p_{1}$ and the upper bound on $z_{1}$ obtained via the $L B_{1}$ computation. The second estimate in strategy (3) is the minimum of $r e p_{2}$ and the upper bound on $z_{2}$ associated with $L B_{1}^{L R}$ and the first estimate in strategy (4) is the minimum of $r e p_{1}$ and the upper bound on $z_{1}$ associated with $L B_{2}^{L R}$. In each setting, only the bounds mentioned are actually computed for fathoming.

Three different initial upper bounds have been tested, each based on a relaxation for the global lower bound (see Table 4). For settings (2) and (3), the upper bound on $z_{1}$ for computing $L B_{2}$ stems from Lagrangian relaxation. The search tree is explored in a depthfirst fashion because best-first and breadth-first do not always find feasible solutions within the time limit. Branching strategy (3) combined with initialization setting (1) achieves the best results: it seems that the exploration of more nodes in the search tree is preferable over computing a lower bound on $z_{2}$ in each node.

\footnotetext{
${ }^{1}$ More concretely $(m, n, \tau)=(36,288,5),(40,320,5),(44,352,6)$ and $(48,384,6)$.
} 


\section{$7 \quad$ Beam search}

Beam search (see, e.g., Bisiani, 1992; Ball, 2011) is a heuristic framework based on a B\&B procedure with a breadth-first strategy, which provides a structured approach to a partial examination of an enumeration tree. The technique systematically develops a low number of solutions in parallel in an attempt to find good solutions with minimal search effort. At the first level of the tree, only the $b$ most promising nodes are retained as nodes to branch from; the parameter $b$ is called the beam width. After determining these $b$ nodes, the algorithm proceeds independently for each node, generating one partial tree from each. We refer to these partial trees as beams. For each beam, one node is selected among the descendants of each node. In this way, we obtain $b$ nodes at each level of the search tree and the search progresses through $b$ parallel beams. The thus-chosen $b$ beams descending the search tree are pursued in a breadth-first fashion until each beam is either fathomed or leads to a complete schedule. Clearly, beam search will tend to require substantially less computational effort than standard B\&B procedures, at the expense of the loss of guarantee of finding an optimal solution and the inability to recover from 'wrong' decisions.

Beam search typically relies on an evaluation function to determine which nodes to explore. This poses the problem of striking a good balance between quick, but poor, evaluation and computationally more involved, but more informative, evaluation. To find a good tradeoff economically and quickly, a two-stage approach usually referred to as filtered beam search can be used. At each level, each node is initially evaluated by a crude but computationally inexpensive filtering procedure. Per beam, only the $f$ best nodes, with $f>b$ the so-called filter width, are shortlisted; the remainder is said to be 'filtered out'. Subsequently, the retained nodes are evaluated more accurately to select the node to continue each beam from. Several articles applying filtered beam search to scheduling problems are available in literature (see, e.g., Ow and Morton, 1988; Sabuncuoglu and Bayiz, 1999; Wang et al., 2008; Fliedner et al., 2011). A graphical illustration is presented as Figure 3, where the explored search trees are shown for an instance with $n=7, m=3$ and $\tau=1$ and without coupled trailers, so there are 14 nodes at level 1 . Whereas the B\&B algorithm would branch on all black nodes of Figure 3(a), beam search only branches on a predefined limited number. In the illustration, the beam width $b=2$ for both the standard and the filtered variant; the filter width $f=4$.

Both standard and filtered beam search have been implemented. The same branching strategies (here: evaluation functions) and the same initial solutions as for the B\&B algorithm are tested for the standard procedure. For the filtered procedure, we limit the combinations to logical choices where the second bound takes more computation time than the first one. Tables 5 and 6 indicate that for the standard procedure, the best algorithmic choices are the same as for B\&B. For the filtered algorithm, the best implementation uses two Lagrangian lower bounds. Some preliminary experiments were used for parameterization (setting the widths).

The major disadvantage of beam search is that pruning a node, in particular a node

leading to an optimal or to nearly optimal solutions, can never be recovered. The recovering beam search method overcomes this issue by introducing a recovering step at each level of the search tree, which searches for improved partial solutions (see, e.g., Della Croce and T'kindt, 2002; Valente and Alves, 2005). This recovery step verifies whether the current 


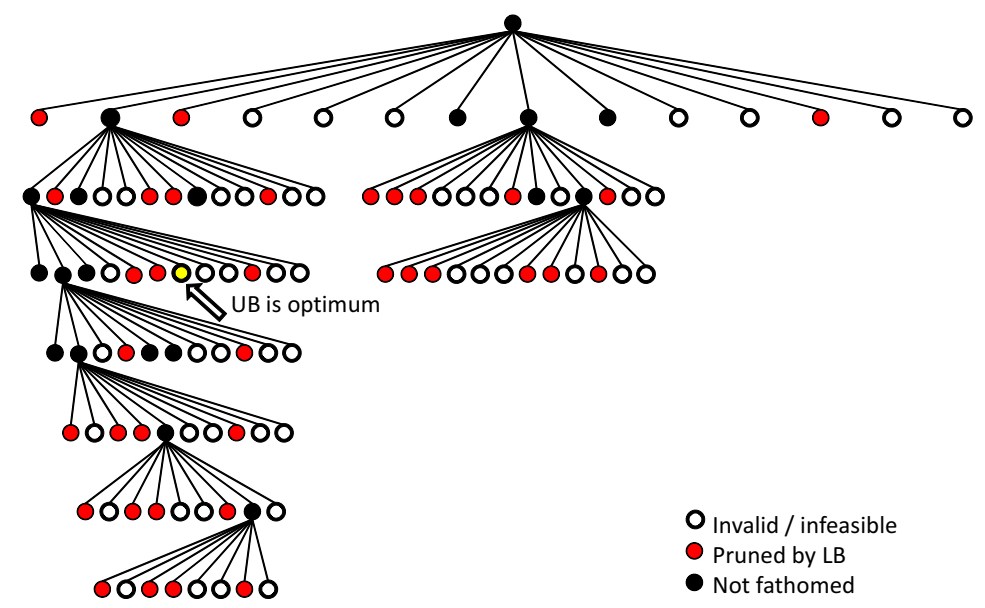

(a) Standard beam search

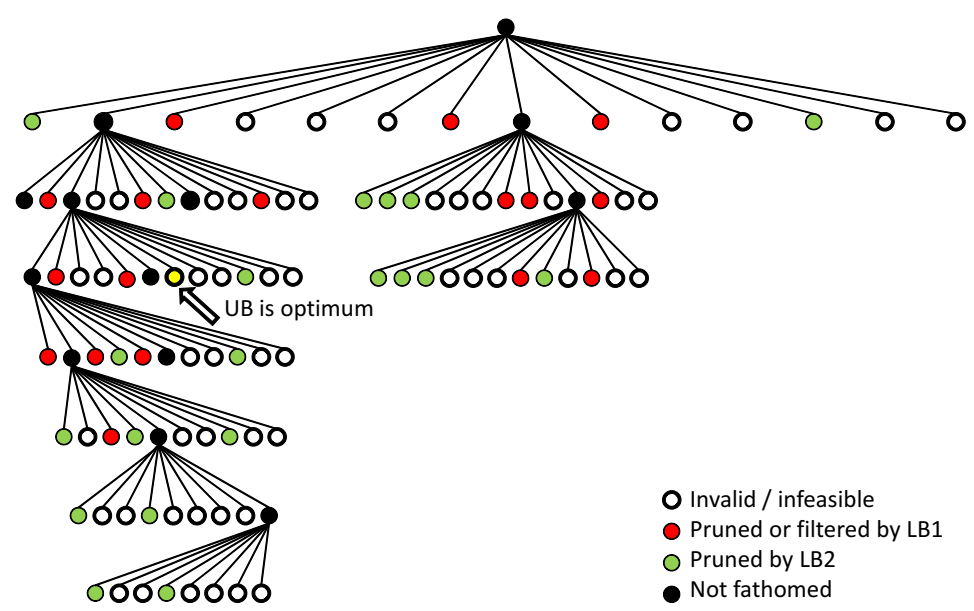

(b) Filtered beam search

Figure 3: Two search trees for a small example.

Table 5: Comparison of different settings for standard beam search.

\begin{tabular}{|l|l||ll|}
\hline & Setting & $z_{1}$ & $z_{2}\left(z_{1}\right)$ \\
\hline \multirow{5}{*}{ Branching strategy } & $(1) L B_{1}^{L R}$ and $L B_{2}^{L R}$ & $17.74 \%$ & $-4.93 \%$ \\
& $(2) L B_{1}^{L R}$ and $L B_{2}^{P M}$ & $58.96 \%$ & $-4.53 \%$ \\
& $(3) L B_{1}^{L R}$ and $U B_{2}$ & $0.00 \%$ & $0.00 \%$ \\
& $(4) U B_{1}$ and $L B_{2}^{L R}$ & $30.70 \%$ & $-5.91 \%$ \\
\hline \multirow{3}{*}{ Initial solution } & $(1) L B_{1}^{L R}$ & $0.00 \%$ & $0.00 \%$ \\
& $(2) L B_{2}^{L R}$ & $0.07 \%$ & $0.00 \%$ \\
& $(3) L B_{2}^{P M}$ & $29.07 \%$ & $-2.10 \%$ \\
\hline
\end{tabular}


Table 6: Comparison of different settings for filtered beam search.

\begin{tabular}{|l|l||ll|}
\hline & Setting & $z_{1}$ & $z_{2}\left(z_{1}\right)$ \\
\hline \multirow{2}{*}{ Branching strategy } & (1) $L B_{1}^{L R}$ and $L B_{2}^{L R}$ & $0.00 \%$ & $0.00 \%$ \\
& (2) $L B_{1}^{L R}$ and $L B_{2}^{P M}$ & $0.00 \%$ & $0.71 \%$ \\
\hline \multirow{3}{*}{ Initial solution } & (1) $L B_{1}^{L R}$ & $0.00 \%$ & $0.00 \%$ \\
& (2) $L B_{2}^{L R}$ & $0.20 \%$ & $0.08 \%$ \\
& $(3) L B_{2}^{P M}$ & $5.52 \%$ & $0.08 \%$ \\
\hline
\end{tabular}

partial solution is dominated by another partial solution at the same level of the search tree (typically by applying interchange operators to the current partial schedule). This modification allows to partially recover from previous wrong decisions and can be seen as a local search on the partial solution. Unfortunately, recovering beam search does not seem particularly suitable for the problem at hand because there are no clear dominance rules. Nevertheless, the hybrid algorithm to be presented in Section 8 does explore other solutions than only those in the beams, and may thereby also undo earlier undesirable decisions.

\section{Tabu search}

Tabu search (see, e.g., Glover, 1989) is a metaheuristic procedure that uses local search to iteratively move from a current solution to a solution in the neighborhood of this solution, until some stopping criterion is satisfied. Each solution has an associated neighborhood, and each solution in this neighborhood is reached from the initial solution by an operation called a move: swapping two random tasks in the permutation and if needed, transforming the obtained permutation into a valid one. At each iteration, we do not explore the complete neighborhood but only construct a predetermined number of solutions from which the best is retained, even if it does not improve the current objective value. In this way, the chance of becoming trapped in local optima that are not globally optimal, is reduced. To prevent the search from cycling and re-visiting the same solutions many times, the (reverse of the) most recent moves are classified as forbidden or tabu. In case a tabu move would result in a very promising solution, however, its tabu classification may be overridden. The aspiration criterion that implements this condition evaluates the improvement in the objective value. When no improvements are achieved for a predetermined number of iterations, a new starting solution is created in which the tasks are listed in a random order.

The same initial solutions as for the B\&B algorithm are implemented. For the same

Table 7: Comparison of different settings for tabu search.

\begin{tabular}{|l|l||ll|}
\hline & Setting & $z_{1}$ & $z_{2}\left(z_{1}\right)$ \\
\hline \multirow{3}{*}{ Initial solution } & (1) $L B_{1}^{L R}$ & $15.33 \%$ & $-2.05 \%$ \\
& (2) $L B_{2}^{L R}$ & $0.00 \%$ & $0.00 \%$ \\
& (3) $L B_{2}^{P M}$ & $14.74 \%$ & $-1.28 \%$ \\
\hline
\end{tabular}


instances as in Section 6, Table 7 shows that an initial solution based on the Lagrangian relaxation for $z_{2}$ gives the best results, and that the performance of the algorithm is rather sensitive to this choice. At the same time, experimentation indicates that diversification by means of new random starting solutions does not significantly improve the results. We therefore look for yet other choices for the initial solution, and the algorithms described in the previous sections can serve exactly this purpose. We focus on B\&B and beam search as sources of an improved initial solution, as it will be observed in the next section that the results produced by standard beam search are better than those obtained by the filtered variant. Figure 4 shows the obtained objective values for different proportions of the total running time allotted to B\&B or to beam search, for the same four instances as before. The remaining running time is available for tabu search. The objective values per instance are reported relative to the best value obtained over the different proportions of running time (the best value is represented as 100\%). We find that the best results are obtained by spending some $20 \%$ of the time on either B\&B or beam search, and subsequently running tabu search for the remainder of the available computation time. We will refer to the resulting methods as hybrid algorithms.

\section{Computational results}

The performance of the algorithms proposed in this paper is compared on a large dataset for one hour of computation time. Note that such CPU times are acceptable at TPCE, since a dock assignment is always made one day beforehand. Table 8 shows the values of $z_{1}$ and $z_{2}$ for the best solution found within the time limit. We also include the results for the IP formulation of Section 4 when the solver is interrupted after one hour. We have evaluated the quality of the solver with parameter settings that emphasize feasibility and focus less on proof of optimality (ILOG 2008); with this new setting, however, CPLEX was not able to find a feasible solution for more instances. Moreover, the objective values found were sometimes worse than with the (initial) balanced setting (equal emphasis on feasibility and proof of optimality). We therefore report the results for the latter setting. For some instances, none of the algorithms is able to find a feasible solution. This, however, need not indicate a global shortcoming of the algorithms: it may well be that a feasible solution simply does not exist - there is no guarantee from the outset that the instances are feasible. For the instances concerned, we therefore subsequently extend the length of the planning horizon $H_{\max }$ to 144 and rerun all the tests. In practical terms, this means that the day will be run with overtime.

Table 8 shows that overall, our best algorithm is the hybrid algorithm that combines B\&B and tabu search. The objective values for this algorithm are, on average, some $3.51 \%$ better than the objective values for the standard tabu search (which is the third best performing); this percentage reduces to $2.52 \%$ for the beam/tabu hybrid. For the instances that were solved to guaranteed optimality with respect to $z_{1}$ using CPLEX, we can compute a 'gap' as $\frac{z_{1}-z^{*}}{z_{1}}$, with $z_{1}$ the objective value for the concerned algorithm and $z^{*}$ the optimal objective value. This gap is $15.53 \%$ for standard tabu search, $11.54 \%$ for the beam/tabu hybrid and $9.81 \%$ for the B\&B/tabu hybrid. The truncated IP model tends to produce the best solutions for those instances for which it finds a feasible solution - which is not the case for 


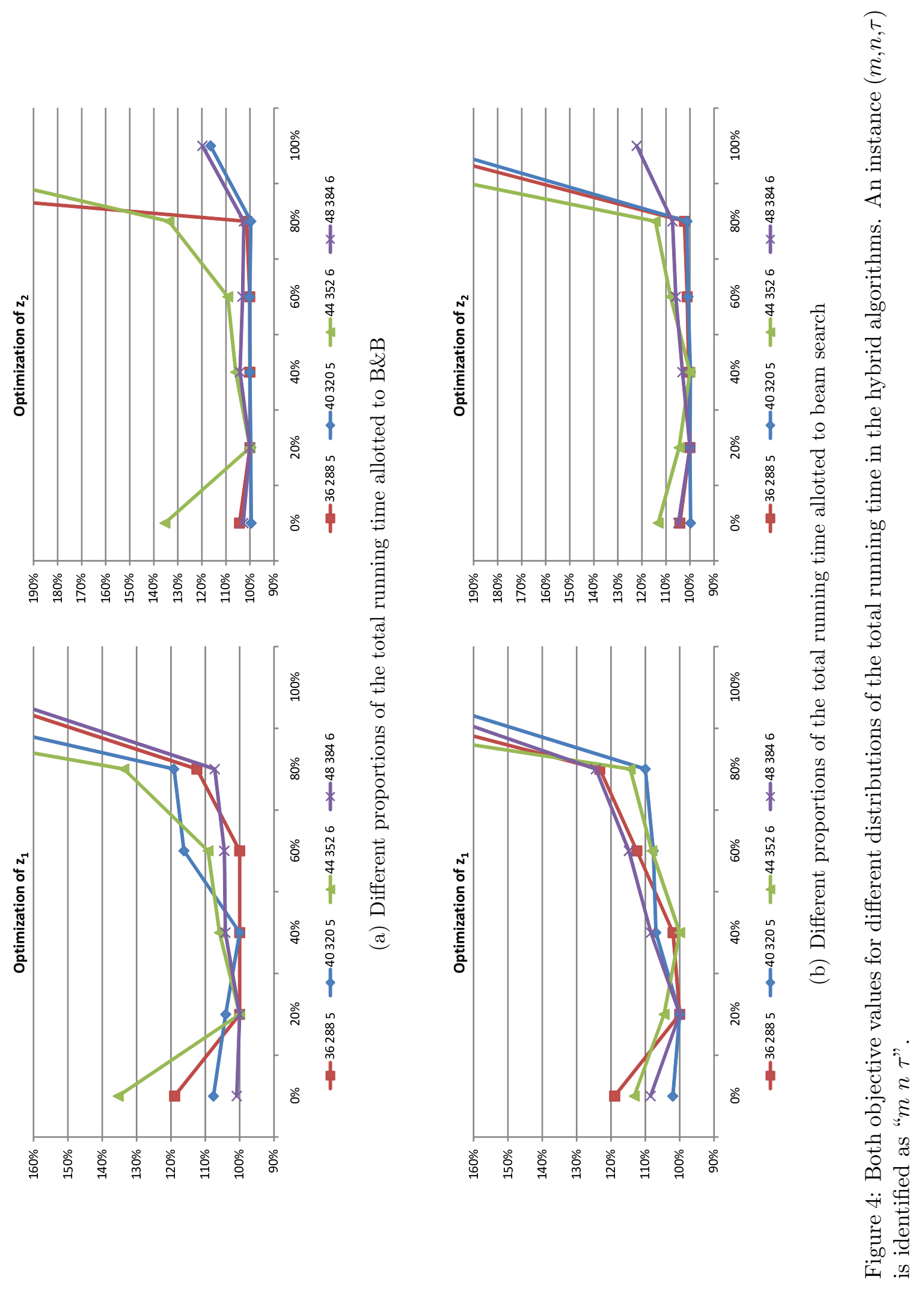




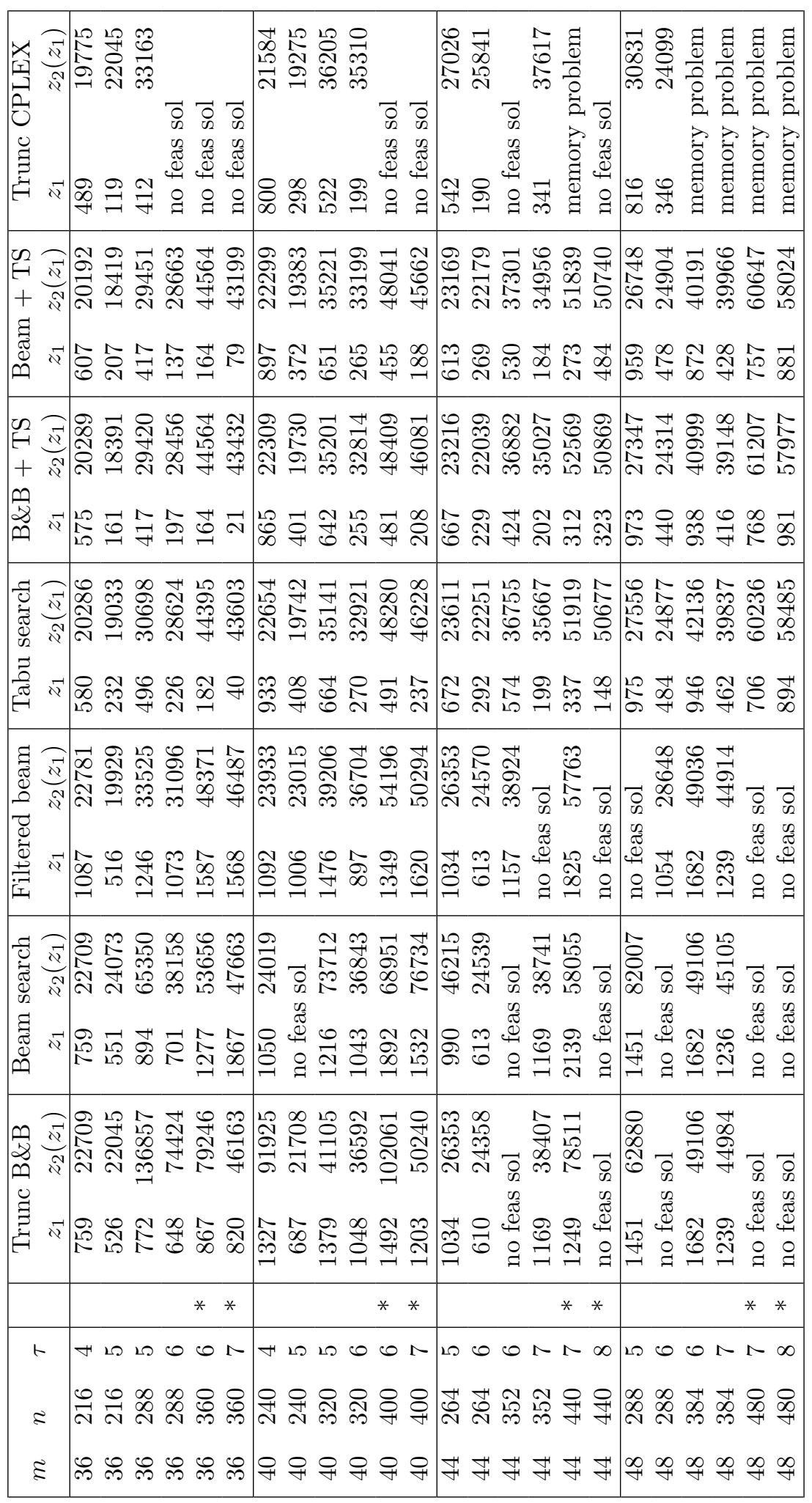


all instances, however.

On a practical note, we need to mention that in spite of the promising computational performance that we are able to report, a practical implementation of our algorithms at the Toyota site seems rather unlikely: our (very enthusiastic) direct contact person at TPCE has left the company, and the interest of the remaining team members responsible for logistics in applying scientific methods for planning, is very low. The recent economic crisis and additional company-related downturns have even further distracted the team's interest. We are convinced, however, that the documented work will be useful to readers confronted with similar planning problems in a practical setting.

\section{Conclusions}

In this paper, we have defined and solved a bi-objective dock assignment problem with trailer transportation, with a clear hierarchy between the two objectives. We have explored the limits of the instance sizes that can be solved to guaranteed optimality within acceptable running times by means of integer programming and branch and bound. It turns out that these limits are too low to be of any use in the practical case that was the prime motivation for undertaking this work, which can require the planning of up to 48 gates, 480 trailers and over 1000 operations. We have therefore also examined the performance of different heuristics for solving large instances, namely a truncated branch-and-bound algorithm, a beam search algorithm, a filtered beam search algorithm and a tabu search algorithm, as well as a hybrid implementation of tabu search with beam search, and with truncated branch and bound. We apply Lagrangian relaxation for computing lower bounds, which can also be transformed into upper bounds (candidate solutions) via a schedule generation scheme. We find that the hybrid algorithms produce the best results, generating high-quality solutions to realistic instances within reasonable computation times. The good performance of the hybrid algorithms is for the larger part achieved by tabu search, but a decent starting solution turns out to be important for the tabu procedure, which is why hybridization is preferable.

\section{References}

G. Alpan, A.-L. Ladier, R. Larbi, and B. Penz. Heuristic solutions for transshipment problems in a multiple door cross docking warehouse. Computers $\&$ Industrial Engineering, to appear.

M. Azizoğlu and O. Kirca. Tardiness minimization on parallel machines. International Journal of Production Economics, 55:163-168, 1998.

M. Azizoğlu and O. Kirca. On the minimization of total weighted flow time with identical and uniform parallel machines. European Journal of Operational Research, 113:91-100, 1999.

K. R. Baker. Introduction to Sequencing and Scheduling. Wiley, 1974.

K. R. Baker and J. C. Smith. A multiple-criterion model for machine scheduling. Journal of Scheduling, 6:7-16, 2003. 
M. O. Ball. Heuristics based on mathematical programming. Surveys in Operations Research and Management Science, 16:21-38, 2011.

L. Berghman, R. Leus, and F. C. R. Spieksma. Optimal solutions for a dock assignment problem with trailer transportation. Annals of Operations Research, to appear.

E. K. Bish, T. Leong, C. Li, J. W. C. Ng, and D. Simchi-Levi. Analysis of a new vehicle scheduling and location problem. Naval Research Logistics, 48:363-385, 2001.

E. K. Bish, F. Y. Chen, Y. T. Leong, B. L. Nelson, J. W. C. Ng, and D. Simchi-Levi. Dispatching vehicles in a mega container terminal. OR Spectrum, 27:491-506, 2005.

R. Bisiani. Beam search. In Encyclopedia of Artificial Intelligence, pages 1467-1568. Wiley Interscience Publication, 1992.

A. R. Boloori Arabani, S. M. T. Fatemi Ghomi, and M. Zandieh. Meta-heuristics implementation for scheduling of trucks in a cross-docking system with temporary storage. Expert Systems with Applications, 38:1964-1979, 2011.

J. Böse, T. Reiners, D. Steenken, and S. Voss. Vehicle dispatching at seaport container terminals using evolutionary algorithms. In Proceedings of the 33rd Hawaii International Conference on System Sciences, pages 1-10, 2000.

N. Boysen and M. Fliedner. Cross dock scheduling: Classification, literature review and research agenda. Omega, 38:413-422, 2010.

N. Boysen, M. Fliedner, and A. Scholl. Scheduling inbound and outbound trucks at cross docking terminals. OR Spectrum, 32:135-161, 2010.

C. L. Chen and R. L. Bulfin. Complexity of single machine, multicriteria scheduling problems. European Journal of Operational Research, 70:115-125, 1993.

F. Della Croce and V. T'kindt. A recovering beam search algorithm for the one-machine dynamic total completion time scheduling problem. Journal of the Operational Research Society, 53:1275-1280, 2002.

M. L. Fisher. The Lagrangian relaxation method for solving integer programming problems. Management Science, 27(1):1-18, 1981.

M. Fliedner, N. Boysen, and A. Scholl. On the part inventory model sequencing problem: Complexity and beam search heuristic. Journal of Scheduling, 14:17-24, 2011.

F. Glover. Tabu search - part 1. ORSA Journal on Computing, 1(3):190-206, 1989.

J. N. C. Gupta, K. Krüger, V. Lauff, F. Werner, and Y. N. Sotskov. Heuristics for hybrid flow shops with controllable processing times and assignable due dates. Computers 8 Operations Research, 29:1417-1439, 2002.

M. Haouari, L. Hidri, and A. Gharbi. Optimal scheduling of a two-stage hybrid flow shop. Mathematical Methods of Operations Research, 64:107-124, 2006. 
H. Hoogeveen. Multicriteria scheduling. European Journal of Operational Research, 167: 592-623, 2005.

ILOG. ILOG CPLEX 11.0 user's manual. ILOG, Inc., 2008. Available online at http://www. decf . berkeley.edu/help/apps/ampl/cplex-doc/.

T. Kis and E. Pesch. A review of exact solution methods for the non-preemptive multiprocessor flowshop problem. European Journal of Operational Research, 164:592-608, 2005.

R. Kolisch. Serial and parallel resource-constrained project scheduling methods revisited: Theory and computation. European Journal of Operational Research, 90:320-333, 1996.

R. Linn and W. Zhang. Hybrid flow shop scheduling: A survey. Computers and Industrial Engineering, 37:57-61, 1999.

Z. Miao, A. Lim, and H. Ma. Truck dock assignment problem with operational time constraint within crossdocks. European Journal of Operational Research, 192:105-115, 2009.

R. H. Möhring, A. S. Schulz, F. Stork, and M. Uetz. Resource constrained project scheduling: Computing lower bounds by solving minimum cut problems. Lecture Notes in Computer Science, 1643:139-150, 1999.

R. H. Möhring, A. S. Schulz, F. Stork, and M. Uetz. Solving project scheduling problems by minimum cut computations. Management Science, 49(3):330-350, 2003.

O. Moursli and Y. Pochet. A branch-and-bound algorithm for the hybrid flowshop. International Journal of Production Economics, 64:113-125, 2000.

R. Nessah, F. Yalaoui, and C. Chu. A branch-and-bound algorithm to minimize total weighted completion time on identical parallel machines with job release dates. Computers $\&$ Operations Research, 35:1176-1190, 2008.

T. Nichi, Y. Hiranaka, and M. Inuiguchi. Lagrangian relaxation with cut generation for hybrid flowshop scheduling problems to minimize the total weighted tardiness. Computers E Operations Research, 37:189-198, 2010.

P. S. Ow and T. E. Morton. Filtered beam search in scheduling. International Journal of Production Research, 26(1):35-62, 1988.

C. D. Paternina-Arboleda, J. R. Montoya-Torres, M. J. Acero-Domingues, and M. C. Herrera-Hernandez. Scheduling jobs on a k-stage flexible flow-shop. Annals of Operations Research, 164:29-40, 2008.

M. L. Pinedo. Scheduling. Theory, Algorithms, and Systems. Springer, third edition, 2008.

I. Ribas, R. Leisten, and J. M. Framiñan. Review and classification of hybrid flow shop scheduling problems from a production system and a solutions procedure perspective. Computers \& Operations Research, 37:1439-1454, 2010. 
I. Sabuncuoglu and M. Bayiz. Job shop scheduling with beam search. European Journal of Operational Research, 118:390-412, 1999.

R. Sadykov and L. A. Wolsey. Integer programming and constraint programming in solving a multimachine assignment scheduling problem with deadlines and release dates. INFORMS Journal on Computing, 18(2):209-217, 2006.

S. C. Sarin and R. Hariharan. A two machine bicriteria scheduling problem. International Journal of Production Economics, 65:125-139, 2000.

S. O. Shim and Y. D. Kim. Scheduling on parallel identical machines to minimize total tardiness. European Journal of Operational Research, 177:135-146, 2007.

R. Soltani and S. J. Sadjadi. Scheduling trucks in cross-docking systems: A robust metaheuristics approach. Transportation Research Part E, 46:650-666, 2010.

L. Tang and H. Xuan. Lagrangian relaxation algorithms for real-time hybrid flowshop scheduling with finite intermediate buffers. Journal of the Operational Research Society, 57:316-324, 2006.

V. T'kindt and J. C. Billaut. Multicriteria Scheduling - Theory, Models and Algorithms. Springer, Berlin, second edition, 2006.

V. T'kindt, J. N. D. Gupta, and J. C. Billaut. Two-machine flowshop scheduling with a secondary criterion. Computers \& Operations Research, 30:505-526, 2003.

J. M. S. Valente and R. A. F. S. Alves. Filtered and recovering beam search algorithms for the early/tardy scheduling problem with no idle time. Computers $\mathscr{6}$ Industrial Engineering, 48:363-375, 2005.

A. Vignier, J. C. Billaut, and C. Proust. Hybrid flowshop scheduling problems: State of the art. RAIRO Operations Research, 33(2):117-183, 1999.

S. J. Wang, B. H. Zhou, and L. F. Xi. A filtered-beam-search-based heuristic algorithm for flexible job-shop scheduling problem. International Journal of Production Research, 46 (11):3027-3058, 2008.

W. Yu and P. J. Egbelu. Scheduling of inbound and outbound trucks in cross docking systems with temporary storage. International Journal of Production Economics, 184: 377-396, 2008. 